JOURNAL OF APPLIED CRYSTALLOGRAPHY

ISSN 1600-5767

\section{NMR Multiplet Interpretation: an Infographic Walk- through. By R. A. Valiulin. de Gruyter, 2019. Pp. x, 166. Price 519.50 EUR, \$600.00 USD, 472.50 GBP. ISBN 978-3- 11-060840-3.}

\author{
Gwendal Kervern*
}

Université de Lorraine, CNRS, CRM2, Nancy, France. *Correspondence e-mail: gwendal.kervern@univ-lorraine.fr

This book, written by Dr Roman A. Valiulin, aims to address an issue often left unattended in the interpretation of ${ }^{1} \mathrm{H}$ NMR spectra of organic compounds. The author's concern originates in the fact that, in many organic synthesis papers where the structure of the synthesized compound is analyzed by NMR, the description of complex proton peak shapes is simply given as 'multiplet', without further details on the actual structure of the aforementioned multiplet.

The goal of this book is to give NMR spectroscopists a systematic graphical tool to fully analyze complex ${ }^{1} \mathrm{H}$ multiplets and extract all of the J-coupling information from even the most complex first-order peak systems.

For this purpose, the author starts with a description of multiplet diagrams in the simplest case of a unique first-order J-coupling of the observed group of protons with one or more neighbouring protons. He uses these diagrams to introduce the concepts of tree diagrams and Pascal's triangle to determine the relative intensities within such multiplets.

The author then uses three cases of complex first-order multiplets to explain his method for the determination of every coupling constant that is involved in the observed peak shape. He also explains how to reconstruct the shape from a J-coupling tree in order to verify the measurements obtained from this method. After a short reminder of the steps and rules enunciated in these examples, the author gives an extensive and progressive set of exercises based on experimental ${ }^{1} \mathrm{H}$ spectra to test the reader on the implementation of the five-point method to measure J-couplings in proton first-order multiplets that is developed in this book.

The author has chosen to discuss neither the physics behind the existence of NMR multiplets nor the chemical features that will influence the strength of scalar couplings. He mentions the existence of higher-order scalar couplings but does not go into the details of their complexity. These choices, however drastic they may seem, make the purpose of this book, as well as the typical reader targeted by such a publication, very clear.

The only regret that I have concerning this otherwise very clear description is that there is a lack of information on the determination of relative intensities within the multiplet: even if it is implicit in the method, it should be said that the segments at the extremities are always set to 1 . And cases such as exercise 33 or 44 , where the strong overlap renders the intensity measurements (and, in a sense, the actual positions of the peaks) slightly trickier, should also be treated with a little more attention.

It should be said that the method developed in these pages is simple and clearly explained, so that anyone making routine NMR measurements on organic compounds will be able to extract all the J-coupling information when their spectra allow such measurements. In other words, when applicable, the method developed in this book is extremely efficient.

And that is where some strong questioning comes into play: applicability. This book presents about 50 examples of ${ }^{1} \mathrm{H}$ NMR multiplets for protons that undergo up to five different J-couplings, and no experimental data whatsoever are presented. All of the exercises seem to be based on experimental spectra, presented on a scale in p.p.m. (which is quite singular in the sense that the rounding of numbers along the scale is not quite as fine as the precision of the scale itself), but no experimental conditions under which those spectra were acquired are given. In order to measure J-couplings, it would help to have a 
scale in hertz, or at least a proton Larmor frequency. The molecules responsible for such patterns are not presented in the solution, nor are the solvents in which they have been dissolved.

This is a matter of concern in the sense that some of the most difficult exercises given at the end of the book seem to arise from very complex systems (a group coupled to six inequivalent protons). One wonders how common such systems are and how often the conditions for such clear multiplets to appear are met. It is quite common in molecules with a large number of protons to have overlap between different chemically inequivalent groups, not to mention the numerous cases of second-order multiplets.

In short, this book offers a very well explained and efficient method for a very specific problem encountered by very specialized readers. 